\title{
Men eating female/healthy foods are judged as more feminine than men eating male/unhealthy foods
}

\author{
J. Bradbury and M. Nicolaou \\ School of Applied Sciences, Queensgate, University of Huddersfield, HD1 3DH, UK
}

Gender-based food stereotypes, such as meat is particularly suitable for men whereas vegetables are female foods, appear to be reflected in differences between men and women in dietary intake. For example, in the UK men are more likely than women to eat meat and meat products and women are more likely to eat low fat dairy products and less likely to not eat any fruit ${ }^{(1)}$. 'Male foods' also tend to be less healthy than stereotypically 'female foods'. The aim of this study was to compare judgements of the masculinity, femininity, and attributes associated with eating a healthier diet, of fictional male and female students eating male/unhealthy foods or female/healthy foods.

Following preliminary research with separate samples of the target population, two dietary profiles were created, one containing male/ unhealthy foods (burger with chips for lunch, pizza and beer for dinner), and the other featuring female/healthy foods (pasta salad and fruit for lunch, rice and vegetables with wine for dinner), which were attributed to a fictional male student (John) and a fictional female student (Sarah), yielding 4 conditions (see Table). Sarah/John was described as being of average height and weight, reasonably physically active, and working part-time as a student helper in the University library. Personality attributes from the short form of the Bem Sex Roles Inventory $^{(2)}$ were used to assess perceived masculinity (10 items, $\left.\alpha=0.931\right)$ and femininity $(10$ items, $\alpha=0.933)$, and 8 items $(\alpha=0.893)$ used previously by Oakes and Slotterback ${ }^{(3)}$ to assess characteristics attributed to people eating healthy or unhealthy foods. All attributes were rated on a 7 -point scale $(1=$ not at all, $7=$ completely $)$ and mean scores calculated to facilitate comparison.

200 university students participated in the study (97 females). The score for masculinity was negatively correlated with the healthy score $(r=-0.628, p<0.0001)$, whereas femininity was positively correlated $(r=0.813, p<0.0001)$. The scores for masculinity, femininity, and healthy were all significantly different by condition (all $p<0.0001$ ). Post-hoc comparisons using the Tukey HsD test indicated that the mean scores for each condition were significantly different from all other conditions, with the exception of males eating female foods $v$ s. females eating female foods for masculinity $(p=0.082)$ and healthy $(p=0.134)$. There was no effect of sex of respondent on masculinity score $(p=0.092)$, femininity score $(0.079)$ or healthy score $(p=0.242)$.

\begin{tabular}{|c|c|c|c|c|c|c|}
\hline \multirow[b]{2}{*}{ Condition } & \multicolumn{2}{|c|}{ Masculinity Score } & \multicolumn{2}{|c|}{ Femininity Score } & \multicolumn{2}{|c|}{ Healthy Score } \\
\hline & Mean & SD & Mean & SD & Mean & SD \\
\hline Male eating male/unhealthy foods $(\mathrm{F}=25 ; \mathrm{M}=25)$ & 5.53 & 0.68 & 2.94 & 0.84 & 3.17 & 0.96 \\
\hline Female eating male/unhealthy foods $(F=24 ; M=26)$ & 5.08 & 0.65 & 3.37 & 0.79 & 2.32 & 0.87 \\
\hline Male eating female/healthy foods $(\mathrm{F}=23 ; \mathrm{M}=27)$ & 3.29 & 0.63 & 4.90 & 0.49 & 5.18 & 0.54 \\
\hline Female eating female/healthy foods $(\mathrm{F}=25 ; \mathrm{M}=25)$ & 3.62 & 0.75 & 5.25 & 0.34 & 5.51 & 0.58 \\
\hline
\end{tabular}

$\mathrm{F}=$ number of female respondents, $\mathrm{M}=$ number of male respondents.

Regardless of whether the fictional student was female or male, if s/he consumed foods and drinks that are considered to be suitable for females and healthy, s/he was judged to be significantly more feminine than if s/he consumed foods and drinks considered to be typically male and typically unhealthy, with no significant difference between male and female respondents. Consumption stereotypes associated with gender may help to explain the observed differences in diet between men and women, and they may hinder attempts to improve men's diets if dietary recommendations are perceived as "markers of emasculation"(4).

1. Henderson L, Gregory J \& Swan G (2002) National Diet and Nutrition Survey: Adults Aged 19 to 64 Years. vol. 1: Types and quantities of foods consumed. London: The Stationery Office.

2. Bem SL (1981) Bem sex role inventory professional manual. Palo Alto, CA: Consulting Psychologists Press.

3. Oakes ME \& Slotterback CS (2004-2005) Curr Psychol 23, 267-277.

4. O’Doherty Jensen K \& Holm L (1999) Eur J Clin Nutr 53, 351-359. 